\title{
The influence of flow maldistribution on the performance of inhomogeneous parallel
} plate heat exhangers

Nielsen, Kaspar Kirstein; Engelbrecht, Kurt; Bahl, Christian R.H.

Published in:

International Journal of Heat and Mass Transfer

Link to article, DOI:

10.1016/j.jheatmasstransfer.2013.01.018

Publication date:

2013

Document Version

Early version, also known as pre-print

Link back to DTU Orbit

Citation (APA):

Nielsen, K. K., Engelbrecht, K., \& Bahl, C. R. H. (2013). The influence of flow maldistribution on the performance of inhomogeneous parallel plate heat exhangers. International Journal of Heat and Mass Transfer, 60, 432-439. https://doi.org/10.1016/j.ijheatmasstransfer.2013.01.018

\section{General rights}

Copyright and moral rights for the publications made accessible in the public portal are retained by the authors and/or other copyright owners and it is a condition of accessing publications that users recognise and abide by the legal requirements associated with these rights.

- Users may download and print one copy of any publication from the public portal for the purpose of private study or research.

- You may not further distribute the material or use it for any profit-making activity or commercial gain

- You may freely distribute the URL identifying the publication in the public portal 
The influence of flow maldistribution on the performance of inhomogeneous parallel plate heat exchangers

K.K. Nielsen, K. Engelbrecht and C.R.H. Bahl DTU Energy Conversion

Technical University of Denmark

Frederiksborgvej 399, DK-4000 Roskilde, Denmark kaki@risoe.dtu.dk 


\title{
The influence of flow maldistribution on the performance of inhomogeneous parallel plate heat exchangers
}

\author{
K.K. Nielsen, K. Engelbrecht and C.R.H. Bahl \\ DTU Energy Conversion \\ Technical University of Denmark \\ Frederiksborgvej 399, DK-4000 Roskilde, Denmark \\ kaki@risoe.dtu.dk
}

\begin{abstract}
The heat transfer performance of inhomogeneous parallel plate heat exchangers in transient operation is investigated using an established model. A performance parameter, denoted the Nusselt-scaling factor, is used as benchmark and calculated using a well-established single blow technique. A sample of 50 random stacks having equal average channel thicknesses with 20 channels each are used to provide a statistical base. The standard deviation of the stacks is varied as are the flow rate (Reynolds number) and the thermal conductivity of the solid heat exchanger material.

It is found that the heat transfer performance of inhomogeneous stacks of parallel plates may be reduced significantly due to the maldistribution of the fluid flow compared to the ideal homogeneous case. The individual channels experience different flow velocities and this further induces an inter-channel thermal cross talk.
\end{abstract}




\section{Introduction}

Microchannel heat exchangers show promise for applications that require high cooling power density. It has been suggested that liquid cooling using a microchannel heat exchanger is the best suited technique for cooling electronics as transistor density continues to increase [1]. Microchannels have also been applied to a wide variety of applications, such as cryocoolers, dehumidifiers, Stirling engines, solar power, electronics cooling and magnetic refrigeration $[2,3,4,5,6,7,8]$.

Despite the high theoretical performance, microchannel heat exchanger performance in actual devices has often been reported in experiments to be lower than expected [9]. Several explanations for the relatively large deviations observed have been suggested, such as whether the fluid continuum assumption breaks down, the influence of surface roughness in the channels etc. In Ref. [9] these issues are reviewed and it is concluded that for incompressible, single phase laminar flows with aqueous fluids no new physical phenomena occur in microchannels. This is supported by careful experiments performed on single-channel tubes and square channel heat exchangers in the microchannel range $[10,11]$.

It was shown that flow maldistribution in fluid manifolds can reduce the microchannel heat transfer performance in a microchannel heat exchanger [12], as channels near the edges of the heat exchanger do not receive as much fluid flow as those in the center. The flow and temperature distributions in two parallel microchannels were studied numerically when obstructions such as bubbles or debris were placed in one of the channels [13]. The outlet temperature profile was shown to be affected by an obstruction in one of the channels, but the accompanying change in heat transfer performance was not quantified.

Another explanation of the observed heat transfer degradation is nonuniform plate spacing [14] in the microchannel stack. As heat transfer performance is inversely proportional to plate spacing, the plate spacing is reduced as much as possible for many applications. As dimensions become smaller the relative manufacturing tolerance can become significant. It was shown that variations in fluid channel heights can dramatically reduce the effective heat transfer in a heat exchanger consisting of a stack of microchannels because larger fluid channels receive a disproportionately high fluid flow while smaller channels are starved for flow [14]. The effect was demonstrated through numerical modeling as well as experiments on passive regenerators. 
It was also shown that thermal cross talk, heat transfer between adjacent fluid channels through channel walls, equalizes temperature differences between fluid channels caused by differences in channels heights. Therefore, cross talk between channels generally reduces the negative impact on heat exchanger performance due to a non-uniform distribution of fluid channels.

This article studies the effects of thermal cross talk in more detail and shows its effects on microchannel heat exchanger performance under transient operation. It will be shown that the effect of the cross talk can vary greatly with geometry, material properties, and operating conditions. We show that a non-uniform microchannel stack can fall into three categories from a thermal modeling standpoint: isolated channels, cross talk dominated, and effectively uniform. In the case of isolated channels, the thermal interaction between the channels is so low in relation to other phenomena that the channels can be modeled as individual isolated channels operating in parallel with an acceptable accuracy. In cross talk dominated operation, a detailed model of a stack of non-uniform flow channels is needed in order to obtain accurate results. In the last category the cross talk is sufficiently high in relation to other heat transport, the heat exchanger can be assumed to be a uniform stack. It is shown that a given microchannel stack can fall into any of these three categories of cross talk depending on the fluid flow rate or thermal conductivity of the solid. A detailed numerical model of a microchannel stack is used to calculate the temperature response when the heat exchanger is subjected to a step change in temperature at its inlet. A technique developed for characterizing heat transfer in porous beds [15] is used to calculate the bulk heat transfer in the entire regenerator and compare it to an ideal microchannel stack with uniform fluid channel heights. All results generated here use a transient response technique that was developed primarily for regenerators rather than steady state heat exchangers such as heat sinks. However, the general conclusions should also apply to heat exchangers in general.

\section{Heat transfer in inhomogeneous parallel plate stacks}

The general problem of determining the heat transfer characteristics of a heat exchanger, here specifically parallel plate stacks, involves thermal conduction in a solid material (of which the parallel plates are made), thermal conduction and convection in a heat transfer fluid in intimate contact with the solid and the heat transfer between the two media. In the idealized case 


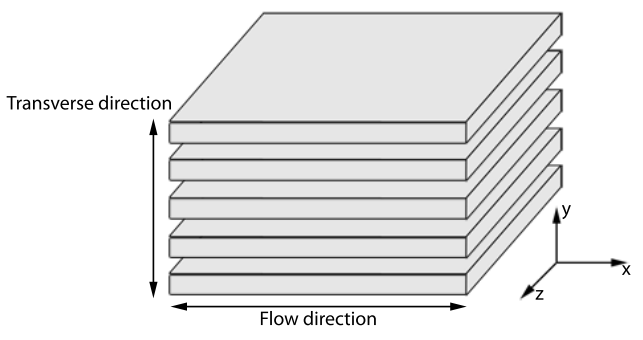

(a)

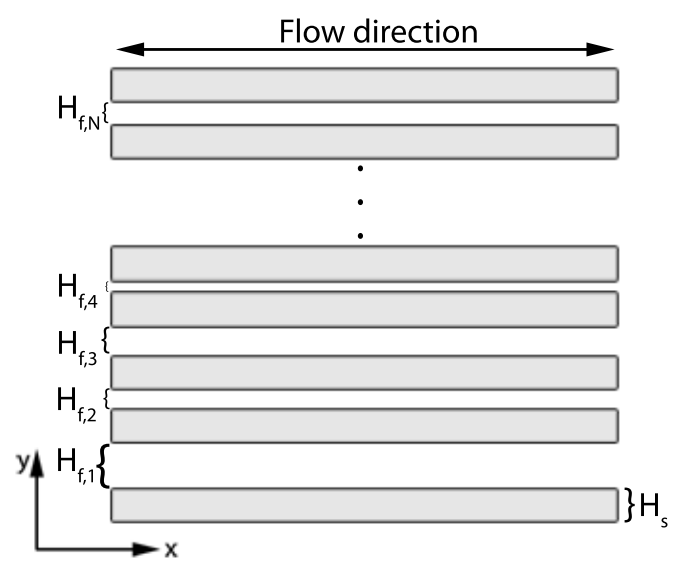

(b)

Figure 1: Schematic of the parallel plate stack geometry. (a) 3D perspective of the stack. (b) the geometry modeled in the detailed model. Each plate has the same thickness whereas the flow channels may have varying thicknesses. The $x$-direction is the direction of the flow and the $y$-direction is referred to as the transverse direction.

the stack is assumed perfectly homogeneous, and it is therefore sufficient to consider half a solid plate and half a fluid channel due to symmetry. Numerical models of such systems have been reported including one-dimensional models where the direction of the flow (or axial direction) is the spatial dimension resolved and, in certain cases, heat transfer transverse to this direction is accounted for via a Biot-Fourier number approach [16]. In other, 2-dimensional, models both the axial direction and the direction transverse to the flow (the $y$-direction in Fig. 1) are numerically resolved for the purpose of studying, e.g., low-thermal conductivity materials $[17,18]$.

It is clear that if the stack is inhomogeneous, e.g., due to varying channel thicknesses and/or plate thicknesses that the symmetries just described will not apply. Each fluid channel will have a different mean fluid velocity, which will cause varying heat transfer in the transverse direction between adjacent channels and plates. This effect is referred to as thermal cross talk. It is non-trivial to provide a general estimate of the influence of this cross talk and in the following section (2.1.3) a term that quantifies the significance of cross talk for a given operating condition is proposed.

The other influence that inhomogeneity in a parallel plate stack has on the heat transfer characteristics is, of course, due to the varying heat transfer conditions in each individual channel caused by the varying flow velocities 
(or flow maldistribution) and thus varying convection coefficients. Larger channels will generally have poorer heat transfer than smaller ones and at the same time significantly larger fractions of the total flow rates thus causing the heat exchanger to perform less efficiently than it would had it been a perfect stack with channel thickness equal to the average spacing of the inhomogeneous stack [14]. These two effects are investigated applying the numerical model described in Sec. 2.1.2 below.

\subsection{Governing equations}

\subsubsection{D homogeneous regenerator equations}

Porous medium regenerators are often modeled as a solid phase interacting with a fluid phase with uniform flow. The geometry of the regenerator is characterized by correlations for pressure drop, heat transfer and other correlations rather than by solving detailed conduction and fluid flow equations to determine them directly. The one-dimensional partial differential equations for the solid and fluid are given below. For the fluid the equation is

$$
\dot{m}_{\mathrm{f}} c_{\mathrm{f}} \frac{\partial T_{\mathrm{f}}}{\partial x}+h A_{\mathrm{HT}}\left(T_{\mathrm{f}}-T_{\mathrm{s}}\right)+\rho_{\mathrm{f}} A_{\mathrm{c}} \varepsilon c_{\mathrm{f}} \frac{\partial T_{\mathrm{f}}}{\partial t}-k_{\mathrm{f}} A_{\mathrm{c}} \frac{\partial^{2} T_{\mathrm{f}}}{\partial x^{2}}=0
$$

where $T$ is temperature, $\rho$ is density, $c$ is specific heat, $k$ is thermal conductivity, $h$, is the heat transfer coefficient, $A_{\mathrm{HT}}$ is the area for heat transfer per unit length, $\varepsilon=\frac{H_{\mathrm{f}}}{H_{\mathrm{f}}+H_{\mathrm{s}}}$ is the porosity, $\dot{m}_{\mathrm{f}}$ is the fluid mass flow rate, $t$ is time and $A_{\mathrm{c}}$ is the cross sectional area. Subscripts s and $\mathrm{f}$ indicate solid and fluid, respectively. The $x$-direction is defined as the direction of the flow (the axial direction; see Fig. 1). The terms represent (in order from left to right) the enthalpy change of the flow, heat transfer from the fluid to the solid, energy storage, and axial conduction in the fluid. Viscous dissipation due to pumping losses is ignored. Eq. 1 cannot directly account for non-homogeneous regenerator geometries because the fluid flow would be a function of the $y$ direction. However, the correlations used to determine heat transfer, pressure drop etc. can be modified to account for non-homogeneous effects.

The $1 \mathrm{D}$ solid material governing equation is

$$
h A_{\mathrm{HT}}\left(T_{\mathrm{f}}-T_{\mathrm{s}}\right)+k_{\mathrm{s}} A_{\mathrm{c}} \frac{\partial^{2} T_{\mathrm{s}}}{\partial x^{2}}=(1-\varepsilon) A_{\mathrm{c}} \rho_{\mathrm{s}} c_{\mathrm{s}} \frac{\partial T_{\mathrm{s}}}{\partial t}
$$

The terms represent heat transfer from the fluid to the regenerator, static axial conduction and energy storage. 
The two equations for a homogeneous regenerator, (1)-(2) can be nondimensionalized by introducing the following terms

$$
\begin{aligned}
t^{*} & =\frac{t}{\tau} \\
x^{*} & =\frac{x}{L} \\
\theta & =\frac{T-T_{\mathrm{C}}}{T_{\mathrm{H}}-T_{\mathrm{C}}} .
\end{aligned}
$$

The characteristic time is $\tau$ and the length of the heat exchanger, $L$, is used as a characteristic length scale. The hot and cold temperatures are denoted $T_{\mathrm{H}}$ and $T_{\mathrm{C}}$, respectively.

Substituting the terms in Eq. 3 into Eq. 1 and 2 and rearranging give the non-dimensionalized fluid equation

$$
\frac{\partial \theta_{\mathrm{f}}}{\partial x^{*}}+\operatorname{NTU}\left(\theta_{\mathrm{f}}-\theta_{\mathrm{s}}\right)+\frac{\Re}{\varphi} \frac{\partial \theta_{\mathrm{f}}}{\partial t^{*}}-\frac{1}{\operatorname{Pe}} \frac{\partial^{2} \theta_{\mathrm{f}}}{\partial x^{*^{2}}}=0
$$

Combining Eq. 1 and 2 and non-dimensionalizing them gives

$$
\frac{\partial \theta_{\mathrm{s}}}{\partial t^{*}}+\varphi \frac{\partial \theta_{\mathrm{f}}}{\partial x^{*}}+\Re \frac{\partial \theta_{\mathrm{f}}}{\partial t^{*}}=\mathrm{Fo} \frac{\partial^{2} \theta_{\mathrm{s}}}{\partial x^{*^{2}}}+\frac{\varphi}{\operatorname{Pe}} \frac{\partial^{2} \theta_{\mathrm{f}}}{\partial x^{*^{2}}} .
$$

The number of transfer units in the regenerator is defined as

$$
\mathrm{NTU}=\frac{h A_{\mathrm{HT}} L}{\dot{m}_{\mathrm{f}} c_{\mathrm{f}}}
$$

and the Peclet number is defined as

$$
\mathrm{Pe}=\frac{\rho_{\mathrm{f}} \tilde{u} L c_{\mathrm{f}}}{k_{\mathrm{f}}}
$$

where $\tilde{u}$ is the average velocity of the fluid. The utilization is defined as

$$
\varphi=\frac{\dot{m}_{\mathrm{f}} c_{\mathrm{f}} \tau}{\rho_{\mathrm{s}} A_{\mathrm{c}} L c_{\mathrm{s}}(1-\varepsilon)},
$$


where the mass flow rate is

$$
\dot{m}_{\mathrm{f}}=\tilde{u} \varepsilon \rho_{\mathrm{f}} A_{\mathrm{c}} .
$$

The ratio of thermal capacity of the fluid and solid domains is

$$
\Re=\frac{\rho_{\mathrm{f}} c_{\mathrm{f}} \varepsilon}{\rho_{\mathrm{s}} c_{\mathrm{s}}(1-\varepsilon)}
$$

Finally, the Fourier number is

$$
\mathrm{Fo}=\frac{k_{\mathrm{s}} \tau}{L^{2} \rho_{\mathrm{s}} c_{\mathrm{s}}(1-\varepsilon)} .
$$

\subsubsection{Inhomogeneous stack model}

A parallel plate heat exchanger is, in the ideal case, straight-forward to analyze accurately in terms of its heat transfer characteristics. However, in the case where the fluid channel thicknesses vary the coupled heat transfer problem of the solid and the fluid may become quite involved. The driving force of this is the asymmetry created by the distribution of channel thicknesses, which generally results in varying fluid velocities in the individual channels - assuming the pressure drop across the heat exchanger is the same for each channel. Larger channels will dominate smaller channels due to their higher flow velocity. This may be seen from the analytical expression for the flow velocities [14]:

$$
\tilde{u}_{i}=\dot{V}^{\prime} \frac{H_{\mathrm{f}, i}^{2}}{\sum_{j=1}^{N} H_{\mathrm{f}, j}^{3}},
$$

where $\tilde{u}_{i}$ is the mean fluid velocity of the $i$ th channel, $\dot{V}^{\prime}$ is the total volumetric flow rate of the stack and $N$ is the number of channels in the stack.

The number of transfer units describing the heat transfer between solid and fluid includes the convective heat transfer coefficient, $h$. This is defined through

$$
\mathrm{Nu}=\frac{h d_{h}}{k_{\mathrm{f}}}
$$

where $\mathrm{Nu}$ is the Nusselt-number and $d_{h}$ is the hydraulic diameter of the heat exchanger. For the present case, flat plates and incompressible heat transfer 
fluid, the Nusselt number is virtually constant and has a value close to 7.54 [22]. The hydraulic diameter is $d_{h}=2 H_{\mathrm{f}}$ with $H_{\mathrm{f}}$ denoting the fluid channel thickness. Due to varying channel thicknesses, $h$ will vary from channel to channel.

A larger fluid channel has a lower heat transfer coefficient (Eq. 13) combined with an increased flow velocity that results in a degradation of the heat exchanger performance. The thermal cross talk may help to restore some of the heat transfer efficiency of the heat exchanger [14].

In order to capture these different effects, it is necessary to apply a detailed numerical model. Such a model was described in Refs. [23, 14] for parallel plate heat exchangers with varying flow channel thicknesses. The geometry modeled is shown in Fig. 1. Thermal conduction is solved for in both the $x$ - and $y$-directions and the fluid convection is included in the flow channel domains. The coupled equations solved for are

$$
\begin{aligned}
\rho_{\mathrm{f}} c_{\mathrm{f}}\left(\frac{\partial T_{\mathrm{f}}}{\partial t}+u_{x} \frac{\partial T_{\mathrm{f}}}{\partial x}\right) & =k_{\mathrm{f}} \nabla^{2} T_{\mathrm{f}} \\
\rho_{\mathrm{s}} c_{\mathrm{s}} \frac{\partial T_{\mathrm{s}}}{\partial t} & =k_{\mathrm{s}} \nabla^{2} T_{\mathrm{s}} .
\end{aligned}
$$

The flow velocity profile is assumed to be fully developed at the channel entrance with a parabolic profile. The model is run with boundary conditions matching a single-blow, which means that the solid and fluid are initially at the same temperature, $T_{\text {init }}$ and the inlet fluid has a temperature $T_{\text {in }}$. In this way the temperature breakthrough curve at the outlet of the stack is recorded as a function of time. An example of such a curve is given in Fig. $2(\mathrm{a})$.

This curve, $T_{\text {out }}(t)$ may be used to analyze the quality of the heat exchanger in terms of its efficiency [15]. Several parameters may be derived from the breakthrough curve that can be used to characterize the heat transfer performance. Here, the time difference between 20 and $80 \%$ of the breakthrough curve are used (see Fig. 2(b) and Refs. [14, 15] for more detail).

As described in detail in Ref. [14] a single-channel model is used to evaluate the heat transfer performance of the inhomogeneous stack. In the single-channel model it is possible to vary the thermal contact between the solid and fluid through a variable contact resistance, $R$. In this way the heat transfer between solid and fluid may be reduced and that leads to a 


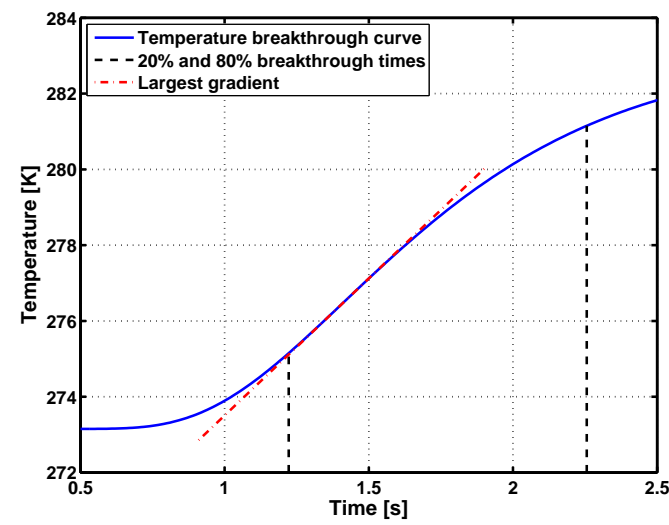

(a)

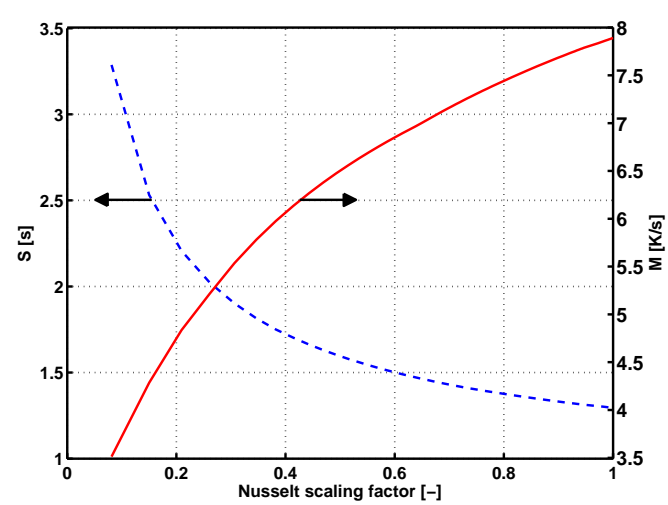

(b)

Figure 2: Example of the analysis scheme applied for evaluating the heat transfer performance. (a) Shows the temperature breakthrough curve as a function of time. The steepets gradient and the time between 20 and $80 \%$ are indicated in the figure. (b) the $S$ (breakthrough time) and $M$ (steepest gradient) values as a function of Nusselt scaling factor (defined in Eq. 16) for a fixed flow rate derived from the single-channel model.

Nusselt-number scaling factor [14]:

$$
\mathrm{Nu}_{\mathrm{scl}}=\frac{1 / h}{1 / h+R}
$$

Figure 2(b) gives the characteristic breakthrough time $(S)$ and the steepest gradient $(M)$ as a function of the Nusselt-scaling factor found at a given flow rate from the single-channel model. In this paper, the detailed model is used to determine the time difference between the 20 and $80 \%$ breakthrough for stacks of varying solid conductivity and channel thickness variation and match those values to an equivalent single channel, or uniformly stacked, heat exchanger model to determine the effective bulk heat transfer coefficient for the entire stack.

It follows from Eqs. 6 and 16 that the NTU for an inhomogeneous stack at a given geometry and flow rate is influenced by the derived Nusselt-scaling factor resulting in an NTU for an inhomogeneous stack:

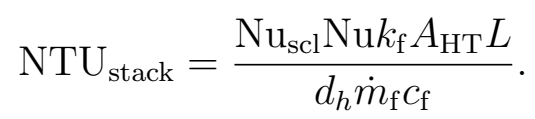

By reducing the inhomogeneous stack down to a homogeneous stack with 
Table I: Central numerical properties / settings used in the simulations.

\begin{tabular}{c|c} 
Description / property & Value \\
\hline Number of gridpoints in the $x$-direction $\left(n_{x}\right)$ & 40 \\
Number of gridpoints in the $y$-direction in each domain $\left(n_{y}\right)$ & 10 \\
Maximum timestep $\left(\Delta t_{\max }\right)$ & $0.01 \mathrm{~s}$ \\
Numerical software & Comsol Multiphysics 4.2 \\
Solver package & PARDISO
\end{tabular}

a reduced NTU value, the complex geometry of non-homogeneous heat exchanger can be modeled in a 1D numerical model, greatly increasing computational efficiency while preserving accuracy. Details on the numerical implementation may be found in Ref. [14]. Table I, however, provides an overview of the important numerical parameters.

\subsubsection{Thermal cross talk between fluid channels}

The non-dimensionalization presented in Eqs. 3-11 is only valid for the idealized case where the heat exchanger is homogeneous and temperature variations transverse to the flow direction can be ignored.

In a homogeneous plate stack, the velocity in each channel is equal and heat transfer from the center of each plate to the fluid is symmetric about its centerline as shown in Eq. 12. When unequal fluid velocities exist at either side of a plate, the heat transfer process becomes more complex. Rather than having equal convection at both sides of the plate, there will be higher convection at one side and the high velocity fluid channel can transfer heat across the plate to the low velocity channel under transient operation. This cross talk process is a complex thermal process that depends on transport properties of the solid, convection at the plate surface, and the time. This means that the fully detailed cross talk model would depend on $F o$ and other parameters as suggested by Eq. 5, and would probably have to be evaluated for a specific operating condition. Characterizing the cross talk in a complete manner would require an analysis such as in Ref. [16] and is outside the scope of the present work. However, the general behavior can be investigated by examining the heat transfer between channels with unequal fluid velocities in channel $i$ as

$$
\dot{Q}_{\mathrm{CT}}=\frac{T_{\mathrm{f}, \mathrm{i}+1}-T_{\mathrm{f}, \mathrm{i}}}{R_{\mathrm{CT}}}
$$

where $R_{\mathrm{CT}}$ is the thermal resistance between two fluid channels. The thermal 
resistance between the two fluid streams will include a convection resistance term, but for this work, $R_{\mathrm{CT}}$ is assumed to be the thermal resistance associated with steady state conduction through the plate:

$$
R_{\mathrm{CT}}=\frac{H_{\mathrm{s}}}{2 A_{\mathrm{HT}} L k_{\mathrm{s}}}
$$

where $H_{\mathrm{s}}$ denotes the plate thickness. If a cross talk term were introduced into Eq. 1 and then non-dimensionalized, the resulting term would be in a form of a number of transfer units cross talk:

$$
\mathrm{NTU}_{\mathrm{CT}}=\frac{1}{R_{\mathrm{CT}} \dot{m}_{\mathrm{f}} c_{\mathrm{f}}} .
$$

When $R_{\mathrm{CT}}$ decreases the thermal cross talk becomes more important and may help to reduce the negative impact on the performance due to flow maldistribution. This happens if the solid thermal conductivity is increased and/or the plate thickness is decreased (see Eq. 19). At large values of $R_{\mathrm{CT}}$ the cross talk becomes negligible and each flow channel may be treated as an individual channel, which makes analysis simpler [19, 20, 21].

\subsection{Parameter variation}

It is clear that not only does the fact that the fluid channels have different thicknesses cause a degradation of the heat transfer performance due to varying flow velocities, but the degree of variation between the individual channel thicknesses may also be very important and the distribution within a given stack likewise. It is, therefore, in order to state anything general (given a certain parameter space in terms of geometry and transport properties) necessary to employ a certain amount of statistics to the analysis.

In real applications the distribution of the channels will generally be random with some overall standard deviation from the mean of the stack. Here, a total of 50 stacks each having 20 channels (and thus also plates) were chosen to provide the base for the analysis. Each stack has the same average channel thickness but the distribution is different for each of the 50 stacks. The individual distributions for each stack were drawn randomly from a normal distribution and the same 50 distributions applied in all cases. The 50 distributions were scaled to keep their mean value but alter their standard deviation, $\sigma$. 
Table II: Fixed parameters used in the detailed model.

\begin{tabular}{c|cccccc} 
Parameter & $c_{\mathrm{f}}[\mathrm{J} /(\mathrm{kgK})]$ & $c_{\mathrm{s}}[\mathrm{J} /(\mathrm{kgK})]$ & $\mu_{\mathrm{f}}[\mathrm{Pa} \cdot \mathrm{s}]$ & $\rho_{\mathrm{f}}\left[\mathrm{kg} / \mathrm{m}^{-3}\right]$ & $\rho_{\mathrm{s}}\left[\mathrm{kg} / \mathrm{m}^{-3}\right]$ & $k_{\mathrm{f}}[\mathrm{W} /(\mathrm{m} \cdot \mathrm{K}$ \\
\hline Value & 4200 & 300 & 0.001 & 1000 & 7900 & 0.6
\end{tabular}

\begin{tabular}{c|ccc} 
& \multicolumn{3}{c}{ Table III: Parameters varied in the detailed model. } \\
Parameter & $\dot{V}^{\prime} / N\left[10^{-6} \mathrm{~m}^{2} \mathrm{~s}^{-1}\right]$ & $\sigma[\%]$ & $k_{\mathrm{s}}[\mathrm{W} /(\mathrm{m} \cdot \mathrm{K})]$ \\
\hline Value range & $2.5-60$ & $5-25$ & $0.5-400$
\end{tabular}

From each stack, given a certain set of operating parameters, the respective $S$ values were derived and the mean value of these found. This final mean $S$ value was then used to derive the Nusselt scaling factor (Eq. 16 and Fig. 2(b)). It was found that using between 40 and 50 stacks gives consistent results whereas only using 20-30 stacks introduces some amount of noise to the results due to an insufficient statistical base.

Clearly, heat exchanger stacks comprise a vast parameter space and only a selected part of this is treated here. Parameters that are kept fixed are given in Table II and those that are varied are given in Table III.

The choice of parameters is based on a heat transfer fluid similar to water and a solid material similar to gadolinium. The product of the mass density and specific heat is very similar when considering metals like gadolinium, aluminum, brass and copper (in the range 2.4 to $3.4 \mathrm{~J} /\left(\mathrm{cm}^{3} \mathrm{~K}\right)$ ). These are common metals used in various heat exchanger applications. Their range of thermal conductivity is somewhat broader and that has been reflected in the choice of the variation of $k_{\mathrm{s}}$; see Tab. III. The variation of the volumetric flow rate, $\dot{V}^{\prime}$, is chosen to give Reynolds numbers ranging from about 5 to 120 .

The Reynolds number is defined as an average value for the stacks for simplicity. It may be expressed as

$$
\mathrm{Re}=\frac{\rho_{\mathrm{f}} 2 H_{\mathrm{f}} \tilde{u}}{\mu_{\mathrm{f}}}=\frac{2 \rho_{\mathrm{f}} \dot{V}^{\prime}}{N \mu_{\mathrm{f}}},
$$

where the dynamics viscosity of the fluid is $\mu_{\mathrm{f}}$ and $H_{\mathrm{f}}$ is the average fluid channel thickness. 


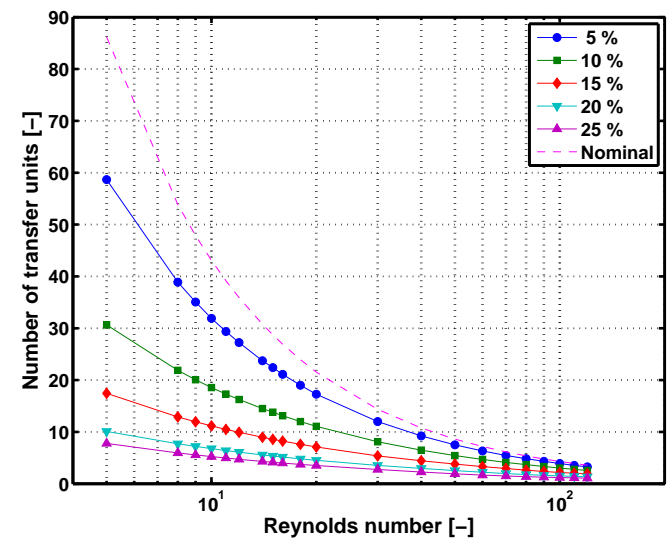

(a)

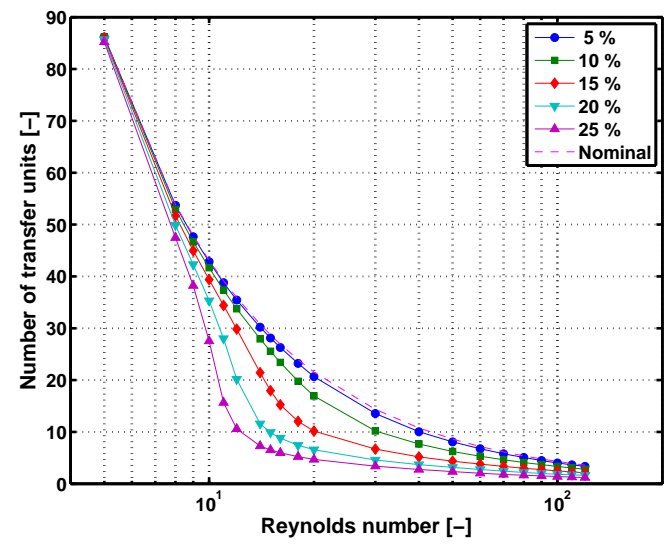

(b)

Figure 3: The effective number of transfer units, $\mathrm{NTU}_{\text {stack}}$, (Eq. 17) as a function of Reynolds number. The dashed line indicates ideal performance (homogeneous stack) and the legend otherwise refers to the standard deviations. (a) the solid thermal conductivity is $10 \mathrm{~W} /(\mathrm{m} \cdot \mathrm{K})$. (b) the solid thermal conductivity is $240 \mathrm{~W} /(\mathrm{m} \cdot \mathrm{K})$.

\section{Results and discussion}

\subsection{NTU as a function of Reynolds number}

The number of transfer units is given as a function of Reynolds number in Fig. 3 for the cases where $k_{\mathrm{s}}=10 \mathrm{~W} /(\mathrm{m} \cdot \mathrm{K})$, similar to gadolinium, and $k_{\mathrm{s}}=240 \mathrm{~W} /(\mathrm{m} \cdot \mathrm{K})$, similar to aluminum, respectively. The trend is threefold. Firstly, the effective NTU is decreased as the standard deviation increases (due to the increased influence of the flow maldistribution). Secondly, at large Reynolds numbers the maldistribution has little overall impact on the NTU since the heat exchanger is overwhelmed by a flow rate too large to sustain sufficient heat transfer (see Eq. 6). Thirdly, at very low Reynolds numbers the maldistribution is seen to have a decreased influence in the case with the larger thermal conductivity of the heat exchanger solid (Fig. 3(b)), whereas for the case with a lower thermal conductivity (Fig. 3(a)) the impact of maldistribution is severe at a standard deviation of $5 \%$ or higher.

These results show that heat transfer performance in non-uniform channels is a complex function of geometry, materials and operating conditions. In cases where the heat exchanger is dominated by axial conduction rather than fluid convection, such as low Reynolds number or large solid thermal conductivity, maldistribution has little impact on performance and the heat 


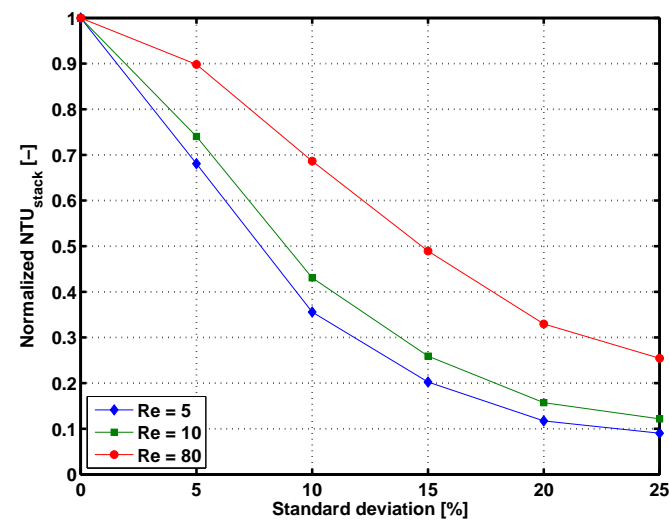

(a)

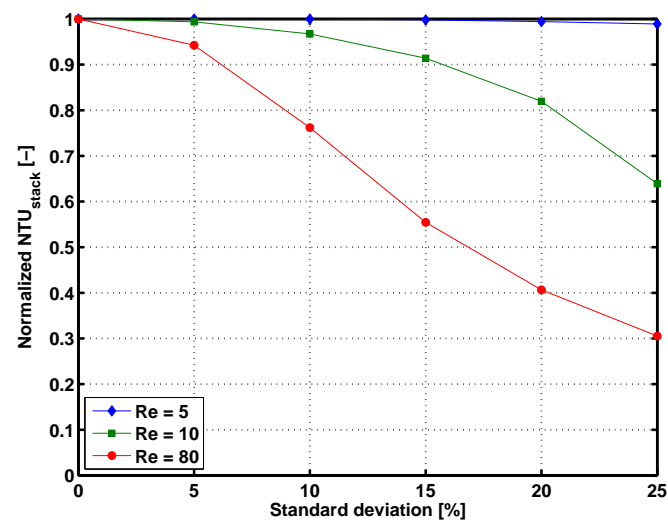

(b)

Figure 4: The effective number of transfer units (Eq. 17) as a function of standard deviation normalized to the zero standard deviation case. The legend refers to the Reynolds number. (a) the thermal conductivity is $10 \mathrm{~W} /(\mathrm{m} \cdot \mathrm{K})$. (b) the thermal conductivity is $240 \mathrm{~W} /(\mathrm{m} \cdot \mathrm{K})$.

exchanger can be modeled as a uniform stack of channels with acceptable accuracy. However, as the Reynolds number is increased for the same geometry, the sensitivity to maldistribution becomes more significant. As an example, consider Fig. 3(b) and a standard deviation of 20\%. When the Reynolds number is around 5 the influence of maldistribution is virtually negligible. However, if it is increased to a value of 20 the effective NTU is reduced to 25 $\%$ of the nominal (ideal) NTU. This effect may explain why heat exchangers in certain cases perform closely to a uniform stack at a lower flow rate while exhibiting performance below expectations as the fluid flow increases.

Considering lower thermal conductivity (Fig. 3(a)) it is seen that even at very low Reynolds numbers the flow maldistribution reduces heat exchanger performance significantly. For the case shown here, even $5 \%$ standard deviation results in a significant deviation from the nominal. The major difference between Fig. 3(a) and 3(b) is thermal conduction between adjacent fluid channels, which is shown to improve overall heat transfer performance.

\section{2. $N T U$ as a function of standard deviation}

The number of transfer units normalized to the ideal case is given as a function of the standard deviation in Fig. 4 for three different Reynolds numbers $(5,10$ and 80, respectively) and two different thermal conductivities (10 and $240 \mathrm{~W} /(\mathrm{m} \cdot \mathrm{K})$, Figs. 4(a) and 4(b), respectively). The results again 
show that the variation in plate spacing can greatly reduce heat transfer performance. It is, perhaps, of greater interest that the performance degradation is a strong function of both the thermal conductivity of the solid and Reynolds number. Performance of the heat exchanger with higher thermal conductivity is less dependent on the standard deviation and shows better performance for all cases modeled than that with lower thermal conductivity. Operating at the larger Reynolds number, however, is seen to result in a performance degradation that depends less weakly on thermal conductivity (as is also indicated in Fig. 3). This indicates that as the conduction between channels is reduced compared to convection to the fluid (a lower value of $\left.N T U_{\mathrm{CT}}\right)$ the heat transfer performance is reduced.

At a large thermal conductivity and small Reynolds number (see Fig. 4(b)) the performance degradation from a high standard deviation is clearly negligible (also seen in Fig. 3(b)). In this case the thermal conduction is a dominating process in the heat exchanger, allowing temperature gradients caused by varying fluid velocities to be smoothed out. Considering the low thermal conductivity (Fig. 4(a)) case it is, however, clear that the performance is degraded the most at the lowest Reynolds number. This is mostly due to the normalization of the NTU, as the lowest Reynolds numbers will result in the highest ideal NTU values, which are most sensitive to variations in fluid flow.

\subsection{NTU as a function of thermal conductivity}

The NTU is given as a function of thermal conductivity at Reynolds numbers of 10 and 80, respectively, in Fig. 5. The plots are normalized to the ideal case for comparison. Again, the trend in heat transfer performance as a function of thermal conductivity does not fit a general form and is dependent on the specific heat exchanger. Considering the high Reynolds number case (Fig. 5(b)) it is seen that the thermal conductivity has relatively little influence on the heat transfer performance and the trend is nearly linear with thermal conductivity. At the higher flow rate, even large solid thermal conductivities do not improve heat exchanger performance markedly. However, the standard deviation is seen to have a significant impact. It may be concluded that in this case a simpler approach than the detailed, 2-dimensional stack model, could be applied for analyzing the heat exchanger performance. Each channel may be treated individually and the final result would be a combination of single channel models. 


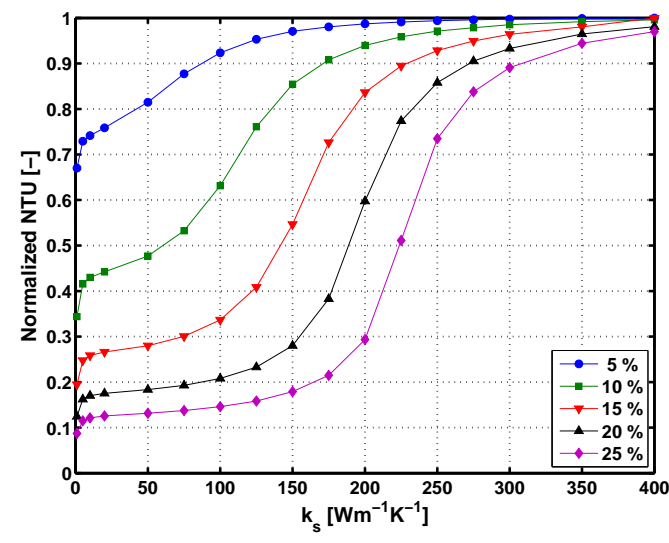

(a)

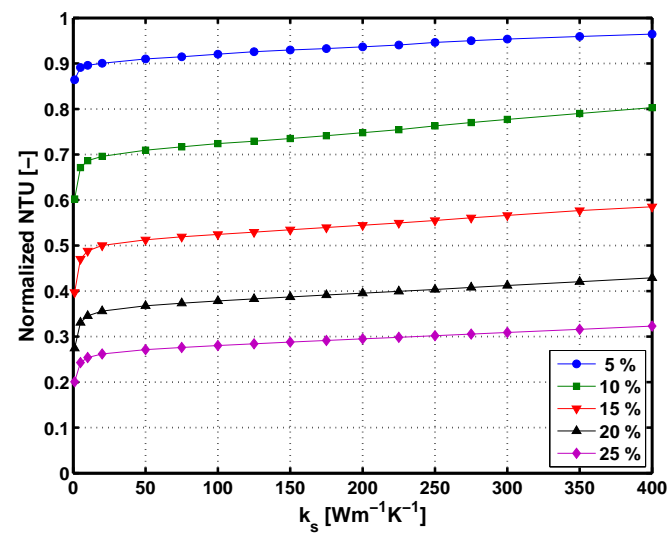

(b)

Figure 5: The effective number of transfer units (Eq. 17) as a function of standard deviation normalized to the zero standard deviation case. (a) the Reynolds number is 10 . (b) the Reynolds number is 80 . The values are normalized to the zero standard deviation case for easier comparison.

However, when considering the lower Reynolds number case of the same heat exchanger (Fig. 5(a)) a significant variation of the NTU as a function of $k_{\mathrm{s}}$ is observed. At large and small values of the thermal conductivity the thermal cross talk has little influence (the graphs in Fig. 5(a) seem to be somewhat S-shaped). There does, however, exist a fairly broad range of intermediate values of the conductivity (from around 75 to, perhaps, 300 $\mathrm{W} /(\mathrm{m} \cdot \mathrm{K})$ in the case considered here) where the thermal cross talk is clearly significant and cannot be ignored or accurately treated with a simpler modeling approach.

\subsection{NTU as a function of cross talk}

The normalized NTU is plotted as a function of $\mathrm{NTU}_{\mathrm{CT}}$ for two plate spacing standard deviations in Fig. 6. For both standard deviation values, the normalized performance increases with increasing $\mathrm{NTU}_{\mathrm{CT}}$. The curves tend to collapse onto themselves when plotted against $\mathrm{NTU}_{\mathrm{CT}}$ as opposed to solid thermal conductivity, suggesting that the $\mathrm{NTU}_{\mathrm{CT}}$ parameter is a sensible variable to consider when evaluating cross talk. Considering Fig. 6(a) when $\mathrm{NTU}_{\mathrm{CT}}$ is below a value of approximately 250 , the heat transfer performance is a weak function of cross talk and the heat exchanger may be treated as a stack of independent channels that do not interact with one another, 


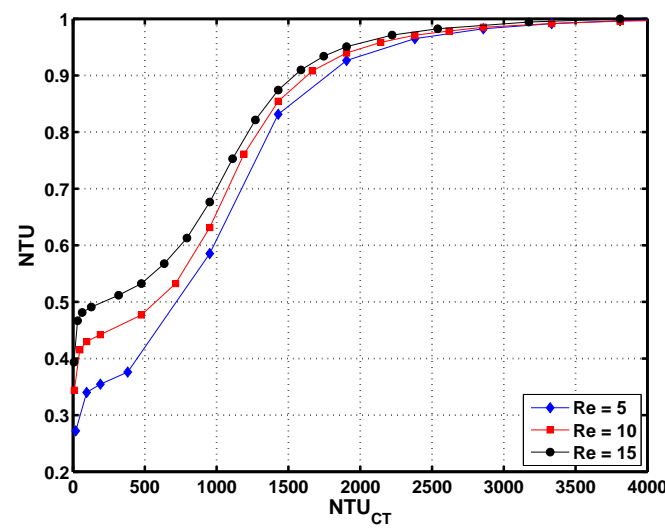

(a)

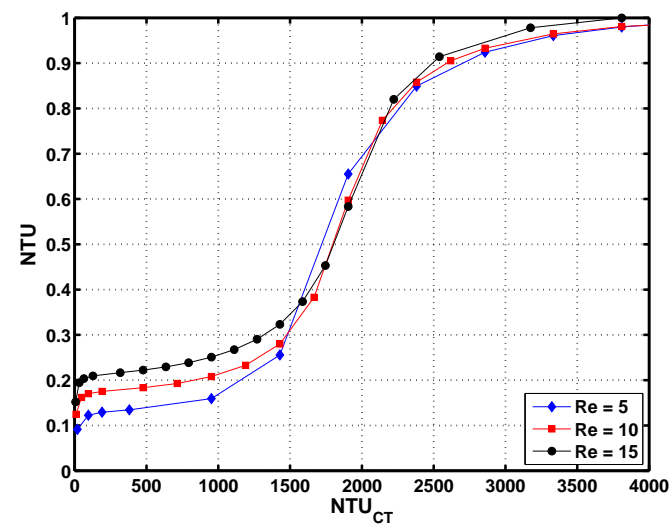

(b)

Figure 6: The effective NTU as a function of $N T U_{\mathrm{CT}}$ (Eq. 20) normalized to the zero standard deviation case at three different Reynolds numbers (indicated in the figure legends). (a) the standard deviation is $10 \%$. (b) the standard deviation is $20 \%$.

thus reducing model complexity. For intermediate values of $\mathrm{NTU}_{\mathrm{CT}}$ ( 2501500) performance is highly dependent on interaction between flow channels and a fully detailed model of the stack is necessary to accurately predict heat exchanger performance. For high values of $\mathrm{NTU}_{\mathrm{CT}}$ (above 1500), variations in flow channel heights have a weak effect on performance and the heat exchanger can be treated as a uniform stack of channels. It should be noted that the values of $\mathrm{NTU}_{\mathrm{CT}}$ shown in Fig. 6(a) are not general and apply only to heat exchangers similar to the one modeled here. Figure 6(b) shows a similar trend as that shown in Fig. 6(a) except the value of $\mathrm{NTU}_{\mathrm{CT}}$ necessary to be considered a uniform stack increases to approximately 2500 because more cross talk between channels is necessary to smooth out temperature variations caused by the larger spacing variation.

One important conclusion to draw from the study of Fig. 6 is that the level of modeling detail necessary to accurately characterize a heat exchanger stack depends on a large number of system parameters, including the operating conditions. A heat exchanger that can practically be considered uniform when operating with a given solid material at a given flow rate may no longer agree with a uniform stack model if the flow rate is increased or it is constructed with a solid with a lower thermal conductivity. Similarly, a given heat exchanger design may begin to deviate from a model that previously accurately predicted performance if the size is scaled down to a level where 
the manufacturing tolerance becomes a significantly larger fraction of the channel height. The term $\mathrm{NTU}_{\mathrm{CT}}$ can be used to give an idea of the significance of the thermal interaction between fluid channels and which type of modeling technique is most appropriate for a heat exchanger.

\section{Conclusion}

It was demonstrated that heat exchangers comprised of inhomogeneous stacks of plates are subject to significant performance degradation over a large span of relevant parameters (Reynolds number, solid thermal conductivity and standard deviation of the parallel plate heat exchanger stacks) compared to uniform plate stacks. Only in cases of large thermal conductivity and low Reynolds number does the effect of flow maldistribution due to stack inhomogeneity become insignificant.

It was shown that the heat exchanger performance may generally be divided into three dominating regimes. At low thermal conductivity the stacks act as a bundle of individual channels that are not communicating thermally (the cross talk is virtually negligible). At elevated conductivities (somewhat depending on standard deviation) small changes in thermal conductivity may lead to significant changes in the performance reduction (caused by the flow maldistribution). Finally, at very large values of the thermal conductivity it was demonstrated that the effect of flow maldistribution is of minor importance and that heat exchangers operating in this regime may be treated as homogeneous stacks.

It is generally concluded that considering heat exchangers built of parallel plates (or similar geometries) may perform significantly less than predicted if using a model that assumes homogeneity in terms of perfect channels (and plates). Even at standard deviations of 5 to $10 \%$ the performance degradation due to flow maldistribution may be severe depending on the Reynolds number at which the heat exchanger is operated and the thermal conductivity of the solid. At larger solid thermal conductivities the effect of flow maldistribution may be reduced.

\section{Acknowledgements}

K.K. Nielsen wishes to thank The Danish Council for Independent Research - Technology and Production Sciences (Contract no. 10-092791) for financial support. 


\section{References}

[1] C. S. Sharma, S. Zimmermann, M. K. Tiwari, B. Michel, and D. Poulikakos. Optimal thermal operation of liquid-cooled electronic chips. International Journal of Heat and Mass Transfer, 55:1957-1969, 2012.

[2] R. Radebaugh. Development of the pulse tube refrigerator as an efficient and reliable cryocooler. Proc. Institute of Refrigeration, 96:11-31, 19992000 .

[3] M. J. White, G. F. Nellis, S. A. Klein, W. Zhu, and Y. Gianchandani. An experimentally validated numerical modeling technique for perforated plate heat exchangers. J. Heat Transfer, 132:111801, 2010.

[4] S. Jain, P. L. Dhar, and S. C. Kaushik. Evaluation of liquid dessicant based evaporative cooling cycles for typical hot and humid climates. Heat recovery systems \& CHP, 14:621-632, 1994.

[5] R. W. Dyson, S. D. Wilson, and R. C. Tew. Review of computational stirling analysis methods. Collect. Tech. Pap. Int. Energy Convers. Eng. Conf., 1:511-531, 2004.

[6] B. Kongtragool and S. Wongwises. A review of solar-powered stirling engines and low temperature differential stirling engines. Renewable $\mathcal{6}$ sustainable energy reviews, 7:131-154, 2003.

[7] R. J. McGlen, R. Jachuck, and S. Lin. Integrated thermal management techniques for high power electronic devices. Appl. Therm. Eng., 24:1143-1156, 2004.

[8] K. A. Gschneidner Jr. and V. K. Pecharsky. Thirty years of near room temperature magnetic cooling: Where we are today and future prospects. Int. J. Refrig., 31:945-961, 2008.

[9] P. Rosa, T.G. Karayiannis, and M.W. Collins. Single-phase heat transfer in microchannels: The importance of scaling effects. Appl. Therm. Eng., 29:3447-3468, 2009.

[10] G. L. Morini. Scaling effects for liquid flows in microchannels. Heat Transfer Eng, 27:64-73, 2006. 
[11] A. G. Fedorov and R. Viskanta. Three-dimensional conjugate heat transfer in the microchannel heat sink for electronic packaging. Int. J. Heat Mass Transfer, 43:399-415, 2000.

[12] J.H. Ryu, D.H. Choi, and S.J. Kim. Three-dimensional numerical optimization of a manifold microchannel heat sink. Int. J. Heat Mass Transfer, 46:1553-1562, 2003.

[13] M. Martin, C. Patton, J. Schmitt, and S. V. Apte. Direct simulation based model-predictive control of flow maldistribution in parallel microchannels. Journal of Fluids Engineering, Transactions of the ASME, 131:1112011-11120117, 2009.

[14] K. K. Nielsen, K. Engelbrecht, D. V. Christensen, J. B. Jensen, A. Smith, and C. R. H. Bahl. Degradation of the performance of microchannel heat exchangers due to flow maldistribution. Appl. Therm. Eng., 40:236-247, 2012.

[15] P.J. Heggs and D. Burns. Single-blow experimental prediction of heat transfer coefficients - a comparison of four commonly used techniques. Experimental Thermal and Fluid Science, 1:243-251, 1988.

[16] K. L. Engelbrecht, G. F. Nellis, and S. A. Klein. The effect of internal temperature gradients on regenerator matrix performance. Journal of Heat Transfer, 128:1060-1069, 2006.

[17] T. F. Petersen, K. Engelbrecht, C. R. H. Bahl, B. Elmegaard, N. Pryds, and A. Smith. Comparison between a 1D and a 2D numerical model of an active magnetic regenerative refrigerator. J. Phys. D: Appl. Phys., 41:105002, 2008.

[18] K. K. Nielsen and K. Engelbrecht. The influence of the solid thermal conductivity on active magnetic regenerators. Journal of Physics D: Applied Physics, 45(14):145001, 2012.

[19] E. U. Schlunder. Mechanism of mass-transfer in heterogeneous systems in particular in fixed-beds, fluidized-beds and on bubble trays. Chemical Engineering Science, 32(8):845-851, 1977. 
[20] W. Brix, M. R. Kærn, and B. Elmegaard. Modelling refrigerant distribution in microchannel evaporators. Int. J. Refrig., 32:1736-1743, 2009.

[21] W. Brix, M. R. Kærn, and B. Elmegaard. Modelling distribution of evaporating co2 in parallel minichannels. Int. J. Refrig., 33:1086-1094, 2010.

[22] M. Nickolay and H. Martin. Improved approximation for the Nusselt number for hydrodynamically developed laminar flow between parallel plates. Int. J. Heat Mass Transfer, 45:3263-3266, 2002.

[23] J. B. Jensen, K. Engelbrecht, C. R. H. Bahl, N. Pryds, G. F. Nellis, S. A. Klein, and B. Elmegaard. Modeling of parallel-plate regenerators with non-uniform plate distributions. Int. J. Heat Mass Transfer, 53:50655072, 2010 . 